\title{
Caudate Nucleus Is Critically Involved in Trace Eyeblink Conditioning
}

\author{
Luke C. Flores and John F. Disterhoft \\ Department of Physiology, Interdepartmental Neuroscience Program, Northwestern University, Chicago, Illinois 60611
}

The basal ganglia are a collection of brain regions involved with motor planning and initiation. The major site of cortical and thalamic input into the basal ganglia network is the striatum, which includes a differentiated caudate nucleus (CN) and the putamen in rabbits. Trace eyeblink conditioning (EBC) is a forebrain-dependent associative learning task in which a stimulus-free time interval separates the presentation of a behaviorally neutral conditioned stimulus (CS) and a behaviorally salient unconditioned stimulus. We investigated whether the $\mathrm{CN}$ is essential for acquisition of trace $\mathrm{EBC}$ and whether learning-related changes in neuronal activity occur in the caudate nucleus during trace EBC. Bilateral lesions of the $\mathrm{CN}$ in rabbits prevent acquisition of trace $\mathrm{EBC}$. In separate cohorts of rabbits, single-unit recordings showed that medium spiny neurons from regions shown to be critical by lesions display strong responses to the CS, especially in the initial days of training before acquisition. Cholinergic interneurons, or tonically active neurons, become responsive to the CS and show dramatic firing rate changes during the trace interval after learning criterion has been met. These data demonstrate that the $\mathrm{CN}$ is required for and involved in trace $\mathrm{EBC}$.

\section{Introduction}

The basal ganglia are involved with motor planning and initiation, as well as learning and memory, particularly "stimulusresponse" or habit memories in which skills are developed gradually after many trials (Packard and Knowlton, 2002). It should be noted that the basal ganglia can be required for performance after just a few trials in certain memory tasks (Broadbent et al., 2007). The role of the basal ganglia, especially the caudate nucleus (CN), in learning and memory has received special interest recently (for review, see Balleine et al., 2009; Packard, 2009; White, 2009).

Eyeblink conditioning (EBC) in rabbits uses a corneal air puff as the unconditioned stimulus (US) and closure of the nictitating membrane of the eye as the unconditioned response (UR) (Gormezano et al., 1962). Work in our laboratory has established whisker deflection as an effective conditioned stimulus (CS) that induces neocortical plasticity in rabbits (Das et al., 2001; Galvez et al., 2006). With repeated pairings of the CS with the US, the rabbits begin to show conditioned eyeblinks (CRs) after CS onset but before US onset. In trace EBC, a stimulus-free temporal gap separates the CS from the US. An intact hippocampus (Solomon et al., 1986; Moyer et al., 1990), caudal anterior cingulate cortex (ACC) (Weible et al., 2000), primary somatosensory barrel cortex (Galvez et al., 2007), and cerebellar deep nuclei (Woodruff-Pak et al., 1985) are essential for acquisition of trace EBC.

Received July 1, 2009; revised Aug. 19, 2009; accepted Sept. 4, 2009.

This work was supported by National Institutes of Health National Research Service Award F31 MH08150801 A2 (L.C.F.) and Grant R01 MH47340 (J.F.D.). We thank Sarah E. Bern for technical assistance during the lesion experiments.

Correspondence should be addressed to Luke C. Flores, Department of Physiology, Interdepartmental Neuroscience Program, Northwestern University, 303 E. Chicago Avenue, Chicago, IL 60611. E-mail: lukeflores2011@u.northwestern.edu.

D01:10.1523/JNEUROSCI.3119-09.2009

Copyright $\odot 2009$ Society for Neuroscience $\quad 0270-6474 / 09 / 2914511-10 \$ 15.00 / 0$
Delay EBC, in which there is no temporal gap between the CS and the US, only requires the cerebellum and brainstem nuclei (Christian and Thompson, 2003); however, the CN may play some role in delay EBC. Lesions of the head of the $\mathrm{CN}$ impair, but do not prevent, acquisition of delay EBC (Powell et al., 1978). Single-unit recordings from the $\mathrm{CN}$ of rabbits during delay $\mathrm{EBC}$ demonstrated that neurons exhibit CR- and UR-related activity during training but rarely respond to the CS (White et al., 1994). Although White and colleagues did not specifically identify the neurons they were recording from, medium spiny neurons (MSNs), which are the GABAergic projection neurons of the striatum, comprise $\sim 90 \%$ of the neurons in the $\mathrm{CN}$, so it is very likely that they recorded from MSNs. Giant cholinergic interneurons, or tonically active neurons (TANs), have been shown to respond differentially to primary rewards and aversive stimuli and shift their responses from the US to the CS during delay conditioning tasks in nonhuman primates (Blazquez et al., 2002; Ravel et al., 2003). Whether these neurons undergo similar learning-related changes during forebrain-dependent trace conditioning has not yet been investigated.

Because trace EBC is an associative learning task that has a clear "stimulus-response" component and is acquired over many trials, it is an excellent candidate for basal ganglia-mediated memory. Furthermore, the $\mathrm{CN}$ receives input from the hippocampal formation (Witter and Groenewegen, 1986), ACC (Weible et al., 2007), and the primary somatosensory SI barrel cortex (Hoffer and Alloway, 2001), which are all necessary for the acquisition of trace EBC.

\section{Materials and Methods}

Subjects. A total of 35 female New Zealand albino rabbits (3-6 months; average weight, $2.9 \mathrm{~kg}$, ranging from 2.2 to $3.9 \mathrm{~kg}$ ) were used in these studies: 25 rabbits for the lesion study and 10 for the recording study. Rabbits were studied because they are excellent subjects for trace EBC and, unlike rodents, have a $\mathrm{CN}$ distinct from putamen, allowing exami- 
nation of its contribution in isolation. These rabbits were individually housed, provided food and water ad libitum, and maintained on a $12 \mathrm{~h}$ light/dark cycle. All behavioral tasks were performed during the light cycle. All surgical and behavioral techniques were performed in accordance with Northwestern University Animal Care and Use protocols.

Recording electrode design and construction. Each tetrode consisted of four Formvar-coated nichrome wires $(25 \mu \mathrm{m}$ diameter bare, $38 \mu \mathrm{m}$ diameter coated) bound together with Epoxylite. The free end of each wire was soldered into a gold-plated Amphenol pin. The tetrodes of each array were passed through a length of 26 gauge hypodermic tubing and glued together. Each electrode array was composed of six tetrodes glued together. Two peripherally mounted drive screws allowed the entire array to be adjusted vertically after implantation.

Surgery. Rabbits were anesthetized with a mixture of ketamine (60 $\mathrm{mg} / \mathrm{kg}$ ) and xylazine $(10 \mathrm{mg} / \mathrm{kg})$. Artificial tears was applied to the eyes to keep them moist. The skull was secured in a stereotaxic apparatus with lambda $1.5 \mathrm{~m}$ below bregma. Under sterile conditions, the skull was exposed and burr holes were drilled to expose implantation sites and to implant anchor screws. Holes were drilled at the following sites relative to bregma [anteroposterior $(\mathrm{AP})+3.5$, mediolateral $(\mathrm{ML}) \pm 2$; $\mathrm{AP}+2, \mathrm{ML}$ \pm 2.2 ; $\mathrm{AP}+1, \mathrm{ML} \pm 2.8]$ for either recording or lesioning electrode implantation. We focused on the head of the $\mathrm{CN}$ because lesions there impaired performance of delay EBC (Powell et al., 1978). For the recording studies, the tetrode was lowered until appropriate neuronal activity corresponding to the $\mathrm{CN}$ was seen (typically between 4.2 and $5 \mathrm{~mm}$ from dura). Dental acrylic was then used to secure the electrode in place. For the lesion experiments, a sharp lesioning electrode was lowered into the caudate nuclei using the same coordinates as the recording experiment. Electrolytic lesions were then made by passing $2 \mathrm{~mA}$ of direct current for $20 \mathrm{~s}$. In sham lesion rabbits, the sharp electrode was lowered into the caudate nuclei but no current was passed. For both the recording and lesioning experiments, dental acrylic was used to build and secure a lightweight head-restraining device containing four nylon bolts to the skull. Rabbits received Buprenex ( 0.3 $\mathrm{mg} / \mathrm{kg})$ immediately after surgery and Metacam $(0.2 \mathrm{mg} / \mathrm{kg})$ immediately after and $24 \mathrm{~h}$ after surgery.

Trace eyeblink conditioning. A total of 24 rabbits underwent trace EBC: seven were used for the recording study, 12 were caudate lesioned, and five were sham lesioned. These rabbits received $1 \mathrm{~d}$ of habituation to the testing chamber and behavioral apparatus. Rabbits were placed in cloth bags and secured in a Plexiglas restrainer inside a sound-attenuating chamber. The right eye was held open with stainless steel dress hooks, and an infrared sensor/air-puff delivery device was attached to the headrestraining device and aimed $\sim 1 \mathrm{~cm}$ from the right eye. The whiskers on the right side of the face were attached to a vibrotactile transducer (T220A4-303; Piezo Systems) using a thin piece of paper and masking tape. The day after habituation, trace EBC began. A computer controlled the delivery of the CS ( $60 \mathrm{~Hz}$ vibration of the whiskers) and the US (3 psi air puff), as well as recorded changes in voltage from the infrared sensor and neural activity from the caudate nuclei when applicable. Each daily session consisted of 80 trials; each trial consisted of a $500 \mathrm{~ms}$ baseline, a $250 \mathrm{~ms} \mathrm{CS}$, a $500 \mathrm{~ms}$ trace period, a $150 \mathrm{~ms}$ US, and a $500 \mathrm{~ms}$ post-US period. Intertrial interval varied between 30 and $60 \mathrm{~s}$, with an average of $45 \mathrm{~s}$. For the lesion study, rabbits were given $10 \mathrm{~d}$ of trace EBC (a total of 800 trials), which is sufficient training to acquire the learning task. For the recording study, rabbits were given $14 \mathrm{~d}$ of training to record neuronal activity from overtrained rabbits.

Pseudoconditioning. A total of 11 rabbits underwent pseudoconditioning: three were for the recording study, four were caudate lesioned, and four were sham lesioned. These rabbits were set up exactly as the traceconditioned rabbits. After $1 \mathrm{~d}$ of habituation, rabbits received daily sessions consisting of 160 unpaired CS-alone or US-alone trials, which varied randomly with an intertrial interval that ranged between 15 and $30 \mathrm{~s}$ with an average of $22 \mathrm{~s}$. These rabbits allowed us to measure the behavioral and neuronal response to unpredictable whisker stimulation and corneal air puffs in a nonlearning situation. In the lesion study, the four caudate-lesioned and four sham-lesioned rabbits were pseudoconditioned for $10 \mathrm{~d}$, after which they received $10 \mathrm{~d}$ of trace $\mathrm{EBC}$ to verify that their caudate nuclei lesions were of appropriate size and location. In the recording study, pseudoconditioning was performed for $14 \mathrm{~d}$.

Delay eyeblink conditioning. We used delay EBC as a necessary control to ensure that the cerebellum and brainstem nuclei required for both delay and trace EBC were intact. All 35 rabbits in this study acquired delay EBC. The rabbits were set up exactly the same as trace-conditioned rabbits. Each daily session consisted of 80 trials: each trial consisted of a $900 \mathrm{~ms}$ CS and a $150 \mathrm{~ms}$ US that began $750 \mathrm{~ms}$ after CS onset and coterminated with the CS. The intertrial interval varied between 30 and $60 \mathrm{~s}$ with an average of $45 \mathrm{~s}$. All rabbits received $4 \mathrm{~d}$ of delay EBC (320 total trials) after initial trace and/or pseudoconditioning.

Behavioral data analysis. For each trial, the baseline voltage was recorded for $250 \mathrm{~ms}$ before CS onset. The movement of the nictitating membrane (third eyelid) during the CS-US interval that resulted in a change $>4$ SDs over pre-CS baseline was considered a CR. CRs that returned to baseline before the onset of the US were considered poorly timed or "non-adaptive" CRs and were not counted for analysis. Any CR that began $<35$ ms after CS onset was considered an "alpha" response and was not counted. Only CRs that resulted in a 4 SD change within 20 ms of US onset were considered appropriately timed "adaptive" CRs. The day in which a rabbit performed eight adaptive CRs in any 10 consecutive trials was considered the day of initial learning in the recording study. To be considered a "learner" in the lesion study, rabbits must have achieved 2 consecutive days of eight adaptive CRs in 10 consecutive trials. Because there were significantly less trials of delay EBC, learning criterion was reduced to $1 \mathrm{~d}$ of eight adaptive CRs in 10 consecutive trials. All rabbits, regardless of previous training or lesion group, achieved learning criterion in delay EBC after $4 \mathrm{~d}$ of training. A group by repeated measures ANOVA was used to determine significant difference between the training groups.

Electrophysiological analysis. Single neuron signals were amplified $(20,000 \times)$, filtered between $600 \mathrm{~Hz}$ and $6 \mathrm{kHz}$, and collected on a personal computer that sampled each channel at $32 \mathrm{kHz}$. Data were collected continuously using Cheetah Data Acquisition System (Neuralynx). Single action potentials were isolated using measurements of spike height and width using Spike Sort Cluster Cutting Tool from Neuralynx, generating two- and three-dimensional scatter plots allowing for tracking the activity of a neuron throughout a single session. Cells recorded from each day were treated as individual neurons because of the possibility of electrode drift between recording sessions. Populations of neurons with similar characteristics were grouped together based on similar firing patterns, interspike interval, and firing rate. TANs in rabbits were differentiated from the more numerous MSNs by using criteria similar to those described in primates (Aosaki et al., 1994). TANs have a baseline firing rate $>1 \mathrm{~Hz}$ (MSNs typically $<1 \mathrm{~Hz}$ ), are tonically active (MSNs typically fire in phasic bursts, followed by long silent periods), have an interspike interval that peaks at $\sim 90 \mathrm{~ms}$ (MSNs peak $\leq 20 \mathrm{~ms}$ ), and show a characteristic pause in firing rate in response to primary stimuli. Perievent timestamp histograms were built to analyze neuronal activity $1 \mathrm{~s}$ before and $2 \mathrm{~s}$ after CS onset using Histogram Builder Tool from Neuralynx. Raster plots were generated using Neuroexplorer (Nex Technologies). To be included in this study, each neuron must have been histologically verified in the caudate nucleus, passed a 2.5:1 signal-to-noise ratio, and had a firing rate sufficient to be analyzed using Wilcoxon's analysis.

Histology. For the recording experiments, marking lesions were made by passing direct current $(50 \mu \mathrm{A})$ for $5 \mathrm{~s}$ through each tetrode on the day before perfusion. Rabbits were anesthetized with a $1 \mathrm{ml}$ injection of Euthasol (390 mg pentobarbital sodium/50 mg phenytoin sodium per milliliter) into the left marginal ear vein and perfused through the heart with saline, followed by $10 \%$ Formalin in saline. The brains were extracted and immersed in 30\% sucrose/10\% Formalin solution until they sank. The brains were blocked and embedded in Tissue-Tek OTC compound, sectioned at $75 \mu \mathrm{m}$ using a freezing stage microtome, mounted on gelatin-subbed slides, stained with cresyl violet and Prussian blue, and coverslipped. A light microscope was used to determine correct electrode placement or to localize caudate lesion damage. $\mathrm{CN}$ lesion pictures were analyzed using NIH ImageJ. Pictures of the $\mathrm{CN}$ were taken at $\mathrm{AP}+4,+3$, $+2,+1$, and +0 , based on a stereotaxic atlas (Girgis and Shih-Chang, 1981). These sites represented the head and rostral body of the CN. The 
A
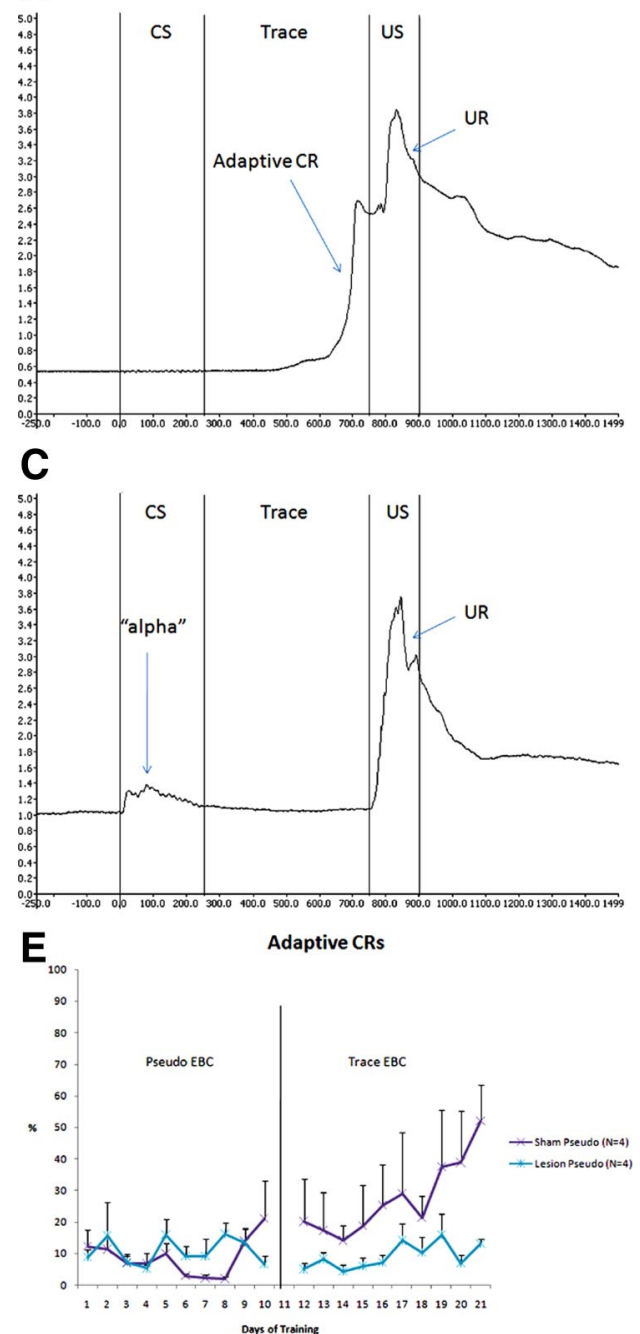

B

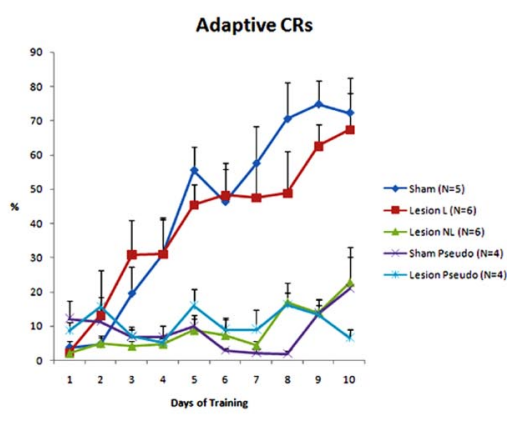

D

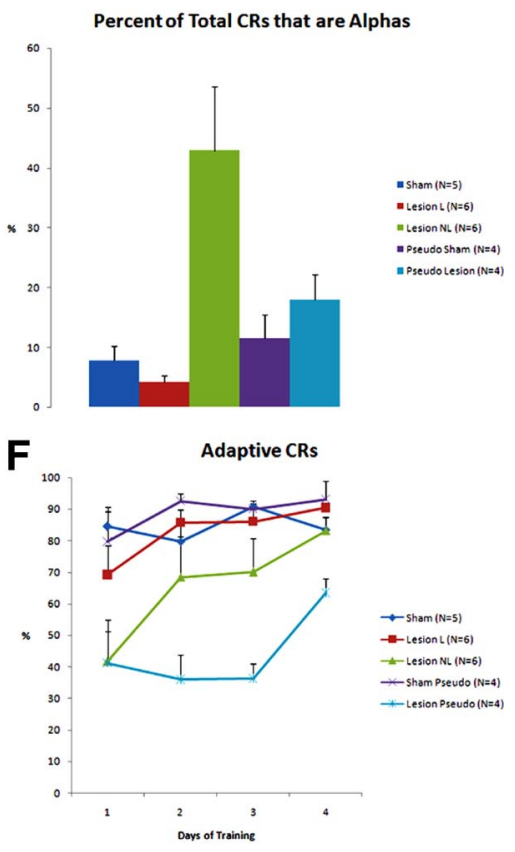

Figure 1. Bilateral lesions of the caudate nucleus prevent adaptive CRs during trace $E B C$ and increase the proportion of nonadaptive alpha blinks. $A$, Example of an adaptive $C R$ in a well trained sham-lesioned rabbit. The $C R$, represented by an increase in voltage from the infrared sensor, is contiguous with the UR, indicating that the nictitating membrane is closing at the time of US onset. $\boldsymbol{B}$, Learning curves for all groups over $10 \mathrm{~d}$ of trace EBC. Sham-lesioned rabbits (Sham) show a steady increase in the percentage of daily trials that resulted in an adaptive CR. Rabbits with small $(<10 \%)$ or strongly unilateral lesions (Lesion $\mathrm{L})$ acquire trace EBC similarly to sham-lesioned rabbits. Sham (Sham Pseudo) and CN-lesioned (Lesion Pseudo) rabbits do not show an increase in adaptive CRs during $10 \mathrm{~d}$ of pseudo EBC, in which the CS and the US are never paired. Rabbits with large bilateral lesions of the CN (Lesion NL) failed to show an increase in the number of adaptive CRs and perform similarly to the pseudoconditioned groups. C, Example of an alpha response. An alpha is a short-latency response that has an onset within $35 \mathrm{~ms}$ of $\mathrm{CS}$ onset. They characteristically have a small amplitude and short duration and are not contiguous with the UR. Note that the rabbit's eye reopens after the alpha response and is open at the time of US onset. D, CN-lesioned rabbits that failed to learn trace EBC showed a high percentage of alpha responses compared with all other groups. None of the other groups were significantly different when compared with each other. $\boldsymbol{E}$, The sham and $\mathrm{CN}_{\mathrm{N}}$-lesioned rabbits that were subject to $10 \mathrm{~d}$ of pseudoconditioning in $\boldsymbol{B}$ were later trained for $10 \mathrm{~d}$ in trace EBC. All sham-lesioned rabbits achieved learning criterion. None of the $\mathrm{CN}$-lesioned rabbits achieved learning criterion. $\boldsymbol{F}$, All rabbits achieved learning criterion ( 8 CRs in 10 successive trials) in non-forebrain-dependent delay EBC. CN-lesioned rabbits that were subject to $10 \mathrm{~d}$ of pseudoconditioning and $10 \mathrm{~d}$ of trace EBC did not reach $80 \%$ total adaptive CRs like the other groups. This is possibly the result of latent inhibition, in which overexposure to the CS and US impairs the rate of conditioning.

average size of the $\mathrm{CN}$ was measured and averaged for all control $\mathrm{CN}$ sections. Percentage of damage was determined for each lesioned rabbit compared with the average control CN size at each landmark.

\section{Results}

Bilateral lesions of the caudate nucleus prevent acquisition of trace EBC

Seventeen rabbits underwent 10 training sessions of trace EBC, each session consisting of 80 CS-US pairings. The CS was a 250 $\mathrm{ms}$ vibration of the whiskers on the right side of the face. The US, a $150 \mathrm{~ms}$ air puff directed toward the right eye, was delivered after a $500 \mathrm{~ms}$ trace period. Adaptive CRs (Fig. 1A), those that occurred within $20 \mathrm{~ms}$ before US onset, were used to evaluate learning. To be considered a learner, a rabbit had to have 2 consecutive days with eight adaptive CRs in any 10 consecutive trials. Figure $1 B$ shows the learning curves for all groups. Repeated measures ANOVA indicated significant differences in the percentage of adaptive CRs between the five groups ( $\mathrm{df}=4, F=25.178, p<0.0001)$. Five rabbits that received sham lesions of the $\mathrm{CN}$ ("sham") acquired trace EBC within $10 \mathrm{~d}$ of training. Six rabbits that received large bilateral lesions of the $\mathrm{CN}$ were unable to acquire trace EBC after $10 \mathrm{~d}$ of training. These CN-lesioned nonlearners ("lesion NL") rabbits performed no differently from sham-lesioned pseudoconditioned rabbits ("sham pseudo") ( $p=$ 0.9421, Fisher's PLSD after repeated measures ANOVA) or CN-lesioned pseudoconditioned rabbits ("lesion pseudo") ( $p=$ 0.7764, Fisher's PLSD after repeated measures ANOVA). There were six rabbits that received lesions of the $\mathrm{CN}$ but learned ("lesion L"), performing similarly to sham rabbits ( $p=0.3324$, Fisher's PLSD after repeated measures ANOVA). The lesion pseudo rabbits did not differ from sham pseudo rabbits $(p=0.6936$, Fisher's PLSD after repeated measures ANOVA).

Figure $1 C$ shows a typical response from a lesion NL rabbit. Although lesion NL rabbits could not display adaptive CRs, they often showed alpha responses, short-latency responses beginning within $35 \mathrm{~ms}$ of CS onset. The six lesion NL rabbits displayed 784 total alphas over $10 \mathrm{~d}$ of training compared with only 169 alphas from the five sham rabbits and 95 alphas from the six lesion L rabbits. They also showed only 1533 total responses compared with 2475 and 2343 responses for the sham and lesion $L$ rabbits, respectively. This resulted in a significantly higher percentage of alpha responses for the lesion NL rabbits compared with all other groups $(\mathrm{df}=4, F=4.718, p<0.05$, Fisher's PLSD after ANOVA). The four lesion pseudo rabbits did not show a significantly higher percentage of alphas during pseudoconditioning than the four sham pseudo rabbits ( $p=0.5753$, Fisher's PLSD after ANOVA); in fact, both groups performed exactly 144 total alphas each over $10 \mathrm{~d}$ of pseudoconditioning. This indicates that the increase in alpha responses occurred as a result of trace EBC.

After pseudoconditioning, the lesion pseudo and sham pseudo rabbits underwent $10 \mathrm{~d}$ of trace EBC. All lesion pseudo rabbits failed to acquire trace $\mathrm{EBC}$, and all sham pseudo rabbits 
acquired trace EBC after $10 \mathrm{~d}$ of training (Fig. $1 E$ ). This indicated that the $\mathrm{CN}$ lesions in the lesion pseudo rabbits were properly located. Overall, 10 rabbits with lesions of the $\mathrm{CN}$ (combined groups illustrated in Fig. $1 B, E$ ) failed to acquire trace EBC. Finally, all CN-lesioned and sham-lesioned rabbits were given $4 \mathrm{~d}$ of forebrain-independent delay EBC, and all achieved learning criterion in delay EBC, regardless of previous training (Fig. $1 F$ ) (supplemental Fig. 1, available at www. jneurosci.org as supplemental material). The caudate-lesioned rabbits were slightly impaired in delay EBC, which is expected based on the findings of Powell et al. (1978) and also possibly a result of latent inhibition.

After training, the rabbits were killed, and their brains were removed and stained with cresyl violet. The lesion NL rabbits all had bilateral damage of the medial and ventral $\mathrm{CN}$, focused particularly from AP +2 to $\mathrm{AP}+3$ (Fig. 2). The lesion $\mathrm{L}$ rabbits had damage that did not exceed $10 \%$ of the $\mathrm{CN}$ and were not focused on the medial or ventral caudate nucleus. One lesion L rabbit (CL17) had significant damage to the medial and ventral left $\mathrm{CN}$, but the right $\mathrm{CN}$ was essentially intact because the lesioning electrode was positioned in the internal capsule (supplemental Fig. 2, available at www.jneurosci.org as supplemental material). This demonstrates that a unilateral lesion was not sufficient to prevent acquisition of trace EBC. The other lesion L rabbits often had damage to the corpus callosum and/or the internal capsule. Despite this damage to white matter and fibers of passage, they were able to acquire trace $\mathrm{EBC}$ normally.

Medium spiny neurons in the caudate nucleus undergo learning-related changes in neuronal activity during trace EBC

Single-unit recordings were taken from the left and right $\mathrm{CN}$ of seven rabbits for $14 \mathrm{~d}$ of trace EBC. In each conditioning trial, the whiskers on the right side of the face were vibrated for $250 \mathrm{~ms}$, and, after a $500 \mathrm{~ms}$ trace period, a $150 \mathrm{~ms}$ puff of air was delivered to the right eye. Three pseudoconditioned rabbits received unpaired presentations of the CS and US. MSNs were differentiated from TANs using interspike interval and firing rate (Fig. 3). MSNs had a peak interspike interval of $17.8 \pm 0.43 \mathrm{~ms}$ and an average firing rate of $0.55 \pm 0.03 \mathrm{~Hz}$. A total of 502 presumptive MSNs (192 from the left $\mathrm{CN} ; 302$ from the right $\mathrm{CN}$ ) were recorded from seven animals over $14 \mathrm{~d}$ of trace EBC. A total of 449 presumptive MSNs ( 213 from the left $\mathrm{CN} ; 236$ from the right $\mathrm{CN}$ ) were recorded from three animals during pseudoconditioning. The overall effect of trace and pseudo EBC on these MSNs is shown in Figure 4, $A$ and $B$. Neurons in both hemispheres showed learning-related
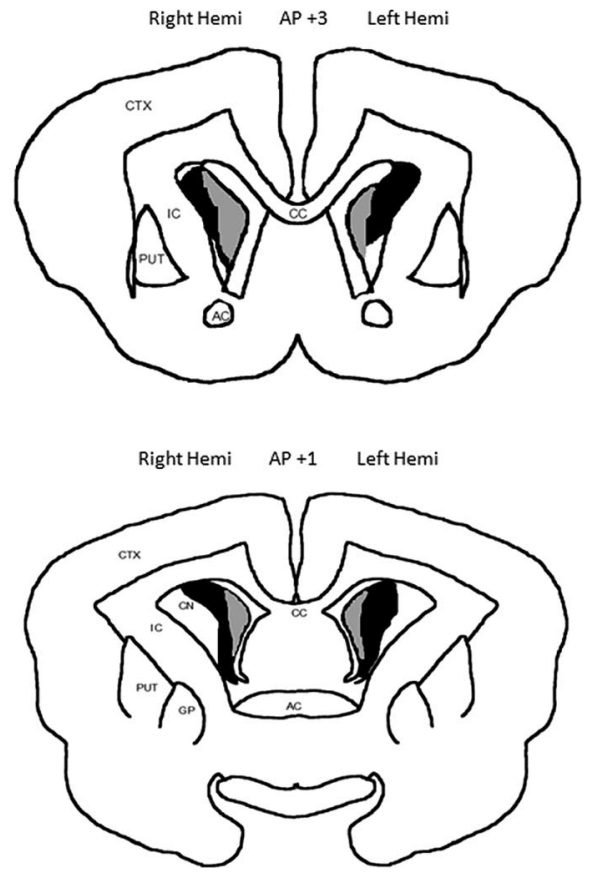

igure 2. Effective lesions included the medial and ventral regions of the caudate nucleus. The smallest (gray) and largest effective lesions of the $\mathrm{CN}$ that prevented acquisition of trace $\mathrm{EBC}$. The smallest effective lesion was bilateral on the medial and ventral CN. AC, Anterior commissure; CC, corpus callosum; CN, caudate nucleus; CTX, cortex; GP, globus pallidus; IC, internal capsule;

A

Figure 3. Criterion for distinguishing MSNs from TANs. A, Representative interspike intervals (ISI) of a presumptive MSN and a TAN (bin size, $10 \mathrm{~ms}$ ). MSNs tend to fire in phasic bursts, giving them an ISI that peaks between 10 and 40 ms and a typically low baseline firing rate. MSNs had an average baseline firing rate of $0.55 \pm 0.03 \mathrm{~Hz}$ with an average ISI of $17.8 \pm 0.43 \mathrm{~ms}$. TANs, despite their tonic activity, have long refractory periods, resulting in a peak ISI between 50 and $150 \mathrm{~ms}$ and typically have a higher versus firing rate (in hertz) differentiates between MSNs (gray triangles) and TANs (black circles). Any cell that exhibited a rightshifted ISI $>50 \mathrm{~ms}$ and a firing rate $>1 \mathrm{~Hz}$ was considered a presumptive TAN. changes in firing rate, although the changes were larger in the left $\mathrm{CN}$ (contralateral to training side) than the right $\mathrm{CN}$ (ipsilateral to training side). There was a clear increase in firing in response to the CS that was absent in pseudoconditioning, indicating that this response to the CS was not to whisker stimulation separate from a learning context. Also, the average baseline firing rate of MSNs was significantly increased in the left $\mathrm{CN}$ during trace EBC compared with pseudo EBC ( $\mathrm{df}=1, F=10.73, p=0.0011$, ANOVA). The increase in baseline firing rate for the right $\mathrm{CN}$ approached significance compared with pseudo $\mathrm{EBC}(\mathrm{df}=1, F=$ $3.582, p=0.0589$, ANOVA). Figure $4 C$ shows the learning curves of the rabbits based around the initial day they performed eight 
A
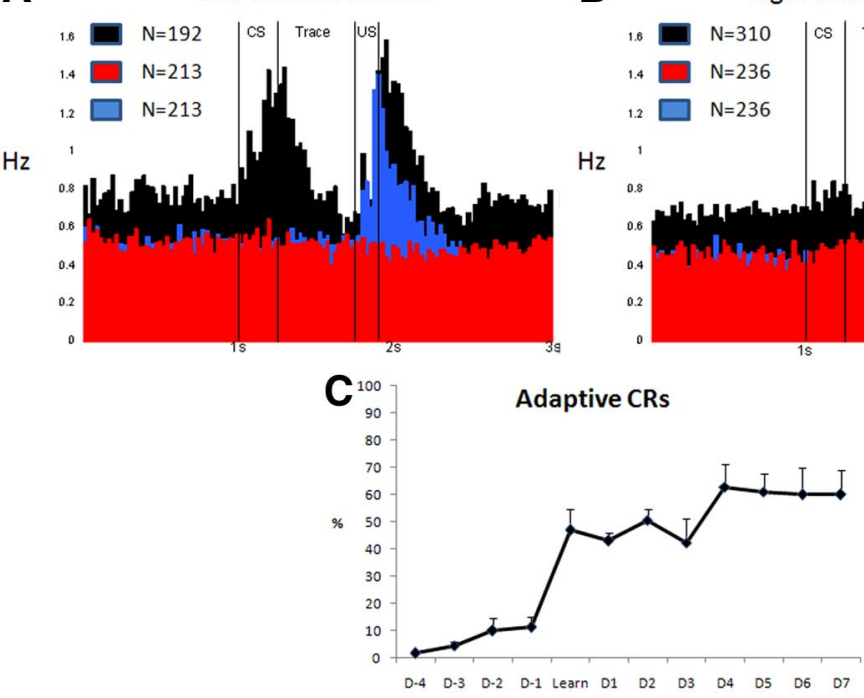

B

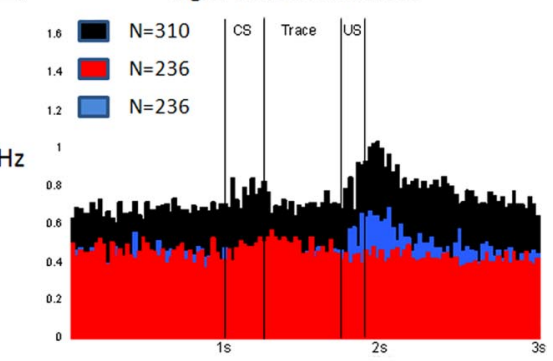

E

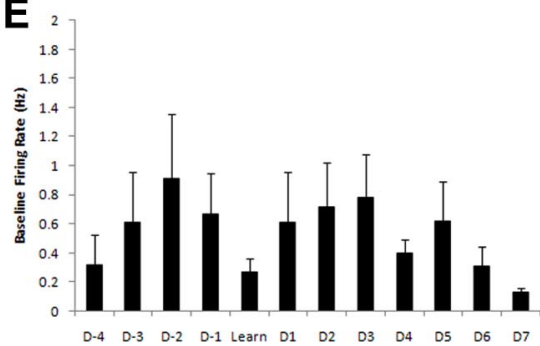

Figure 4. Learning-related changes in the firing of MSNs bilaterally during trace EBC. $A$, Perievent timestamp histograms of the activity of MSNs across all $14 \mathrm{~d}$ of trace (black bars), CS trials of pseudo EBC (red bars), and US trials of pseudo EBC (blue bars) in the left (contralateral to training) CN. There is a significant increase in baseline firing rate in trace-conditioned MSNs compared with pseudoconditioned MSNs in the left $\mathrm{CN}(\mathrm{df}=1, F=10.73, p=0.0011$, ANOVA) and a strong response to the $\mathrm{CS}$ that is absent in CS trials of pseudoconditioning. Bin size of $25 \mathrm{~ms}$. B , Perievent timestamp histograms of the activity of MSNs across all $14 \mathrm{~d}$ of trace (black bars), CS trials of pseudo EBC (red bars), and US trials of pseudo EBC (blue bars) in the right (ipsilateral to training) CN. The increase in baseline firing rate during trace $\mathrm{EBC}$ is not significant compared with pseudoconditioning $(\mathrm{df}=1, F=3.582, p=$ 0.0589 , ANOVA), but there is still a response to the (S and US that is larger than seen in pseudo EBC. These changes are more modest than what is seen in the left CN. Bin size of $25 \mathrm{~ms}$. C, The learning curve for all seven rabbits centered on the day of initial learning ( 8 adaptive (Rs performed in any 10 consecutive trials). There was no statistical difference in the learning curves whether electrodes were implanted in the left $\mathrm{CN}_{\text {, right }} \mathrm{CN}$, or both. $\boldsymbol{D}$, The baseline firing rate for MSNs in the left $\mathrm{CN}$ increases in an orderly manner, becoming significant ( $p<0.05$, Fisher's PLSD after repeated measures ANOVA) in the $2 \mathrm{~d}$ before achieving learning criterion. $\boldsymbol{E}$, The baseline firing rate for MSNs in the right CN does not increase in the same orderly manner compared with the left $\mathrm{CN}$.

adaptive CRs in 10 consecutive trials, constructed to determine whether the increase in baseline firing rate was related to the behavioral learning curves. In Figure $4 D$, the baseline firing rate of MSNs in the left CN steadily increased starting from $4 \mathrm{~d}$ before learning criterion $(\mathrm{D}-4)$, reaching a maximum in the $2 \mathrm{~d}$ immediately before learning criterion ( $\mathrm{D}-2$ and $\mathrm{D}-1)$, before dropping back to initial levels on the day of learning criterion (Learn) and the $7 \mathrm{~d}$ afterward. In Figure $4 E$, the baseline firing rate changed in the right $\mathrm{CN}$, but there was not an orderly increase in firing rate as seen in the left $\mathrm{CN}$.

Regions that undergo learning-related changes correspond to regions that are necessary for acquisition of trace $\mathrm{EBC}$

Recordings made from various regions of the head and body of the $\mathrm{CN}$ during trace EBC were analyzed separately to determine whether there were regional differences in neuronal activity during trace EBC. Not all recording sites in the left $\mathrm{CN}$ showed learning-related changes in MSN activity during trace EBC (Fig. 5). Rather, there appeared to be discrete regions that showed significant CS-, trace-, and US-related activity, especially the medial and ventral $\mathrm{CN}$. It is also clear that these activity patterns occurred at different time points in different regions. Some recording sites in the medial and ventral CN show CS- and US-related activity as early as the first day of training. Other recording sites in the medial and ventral $\mathrm{CN}$ showed strongest responses to the CS and US in the days before learning criterion. The dorsolateral $\mathrm{CN}$ did not show much responsivity at any time during trace EBC.

The recording sites in the right $\mathrm{CN}$ did not show as strong or as clear responses to the CS and the US as the left CN (Fig. 6). Recording site $\mathrm{C}$ in the medial $\mathrm{CN}$ showed the most impressive activity, responding to the CS and US on the initial day of training and on the day of learning criterion. Other recordings sites were either erratically responsive or nonresponsive to trace EBC. During pseudoconditioning, MSNs were responsive to the US (Fig. 7). MSNs in the left CN (contralateral to the eye that the air puff was delivered to) were more responsive to the US than the right $\mathrm{CN}$. There was little response to the CS in either the left or the right $\mathrm{CN}$ during pseudoconditioning.

\section{Tonically active neurons undergo learning-related changes in neuronal activity during trace EBC}

TANs were differentiated from the more numerous MSNs by their interspike interval profile and baseline firing rate (Fig. $3 A)$. TANs had an average baseline firing rate of $3.55 \pm 0.39 \mathrm{~Hz}$ and a peak interspike interval of $85.2 \pm 4.76 \mathrm{~ms}$. TANs were recorded from both the right $\mathrm{CN}$ before and after acquisition of trace $\mathrm{EBC}$, and left $\mathrm{CN}$ after acquisition of trace EBC. In the right $\mathrm{CN}$, there was a very small and brief pause in firing in response to the CS and the US, with a large rebound in firing rate seen after the US (Fig. 8A). After learning, the pause in response to the CS and the US became sharper, as did the rebound firing after the US (Fig. $8 \mathrm{~B}$ ). Individual raster plots show that some TANs in the right $\mathrm{CN}$ were quite responsive to the CS and the US, whereas others remained responsive to only the US. After learning, TANs in the left $\mathrm{CN}$ showed a very steep and long-lasting pause to the CS, a sharp rebound in firing rate during the trace period, and a smaller pause in response to the US (Fig. 8C). The TANs recorded in the left $\mathrm{CN}$ remained responsive to the CS for at least $9 \mathrm{~d}$ after learning criterion was achieved (Fig. $8 C)$. All TANs recorded from the left $\mathrm{CN}$ showed large responses to the CS and US after learning criterion was met. This pattern was unlike the MSNs, in which learning-related changes had primarily diminished at this point in training.

TANs were also recorded from the right and left $\mathrm{CN}$ during pseudoconditioning. There is no response to the CS in either the right or left $\mathrm{CN}$ and a pause in firing in response to the US in both the right and left $\mathrm{CN}$ (Fig. 8D). 


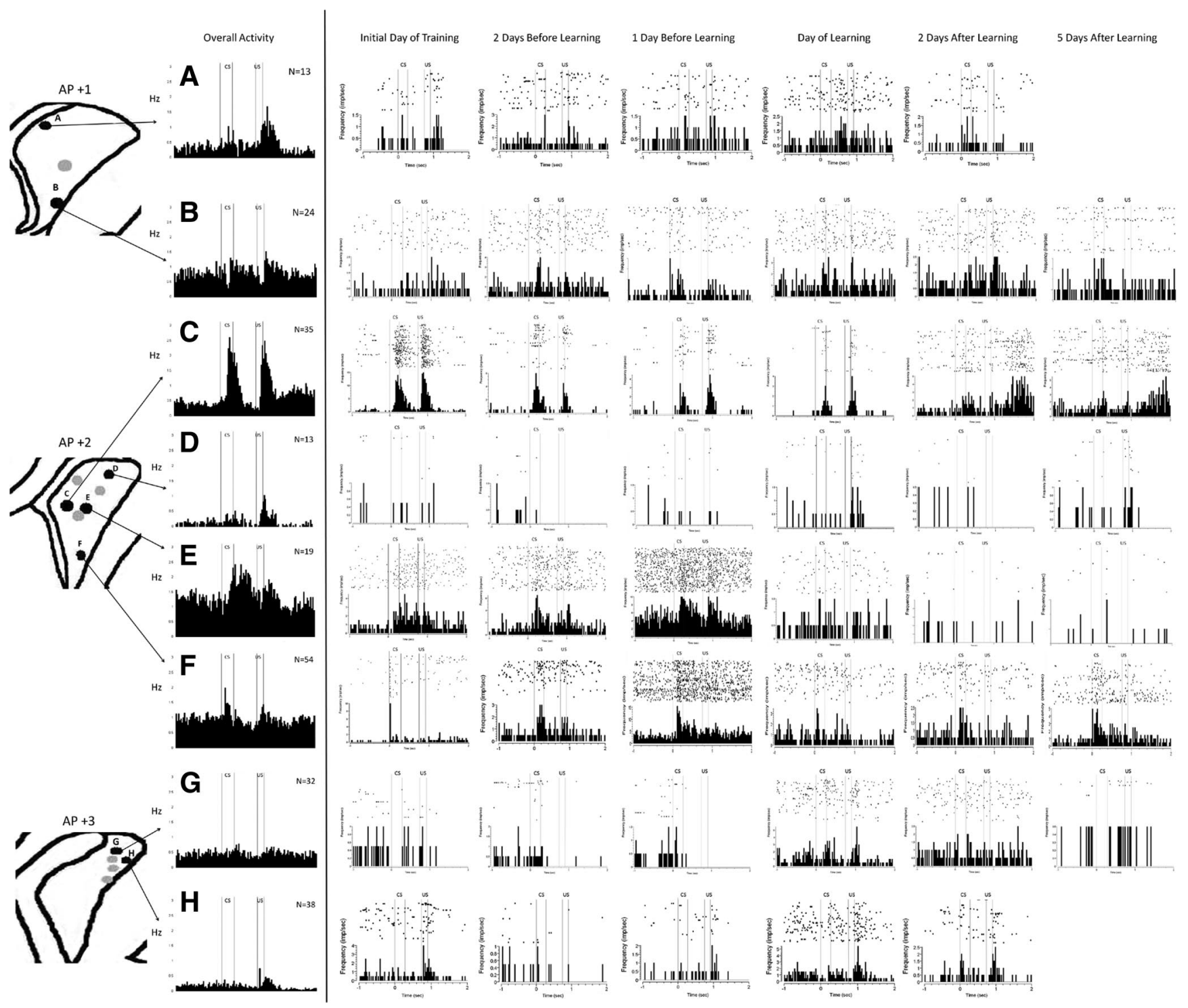

Figure 5. MSNs undergo learning-related changes in discrete locations of the left caudate nucleus. Single-unit recordings from several regions of the left $C N$ during trace $E B C$ (black dots). The most impressive overall activity is seen in medial and ventral regions of the CN at approximately AP +2 . Raster plots of individual neurons show that CS-and US-related activity begins as soon as the initial day of training in certain regions of the $\mathrm{CN}$, especially region C. Other regions, such as $\mathrm{B}, \mathrm{E}$, and F, show their greatest response to the $\mathrm{CS}$ and US in the days before learning. Whereas region $\mathrm{E}$ stops responding with continued training, regions $B$ and $F$ continue to be responsive to the $C S$ for $5 \mathrm{~d}$ after learning. Region $A$ appears to only be responsive to the US. Regions $D, G$, and $H$ in the dorsolateral CN do not appear to be responsive during trace EBC.

\section{Discussion}

These results indicate that the $\mathrm{CN}$ is necessary for acquisition of trace $\mathrm{EBC}$ and that neurons in the $\mathrm{CN}$ undergo rapid and dynamic changes in neuronal firing both before and after learning criterion is reached.

We made bilateral electrolytic lesions of the head and body of the $\mathrm{CN}$ in several rabbits. When the lesions were sufficiently large and included the medial and ventral head and body of the $\mathrm{CN}$, the rabbits failed to acquire trace EBC even after $10 \mathrm{~d}$ of training (800 trials), but were subsequently able to learn forebrainindependent delay EBC within $4 \mathrm{~d}$ of training (320 trials). Damage to fibers of passage is a concern when performing electrolytic lesions. There were six rabbits that had little damage to the $\mathrm{CN}$, often because the lesioning electrode was poorly placed and inadvertently damaged the corpus callosum and/or the internal capsule. These rabbits were unimpaired in trace or delay EBC despite this damage to major white matter tracts. Corticostriatal inputs into the $\mathrm{CN}$ travel along the internal capsule and enter the $\mathrm{CN}$ near their point of termination (for a nice example of single corticostriatal projections, see Parent and Parent, 2006), so electrolytic damage to these fibers blocks afferent input to the medium spiny neurons on which they terminate. In addition, damage to regions of $\mathrm{CN}$ that do not appear to change during trace EBC (Figs. 5, 6) were ineffective, although they obviously damaged both the medium spiny neurons and all fibers of passage traveling within them. Together with our results from the singleunit recording experiment showing that MSNs within the smallest effective lesion showed the earliest and most significant changes in firing rate, this suggests that our learning deficit was a result of neuronal destruction as opposed to damage to fibers of passage.

The impaired lesioned rabbits also exhibited a high percentage of alpha responses, which are a specific early-onset form of shortlatency blinks in response to the conditioned stimulus that have 


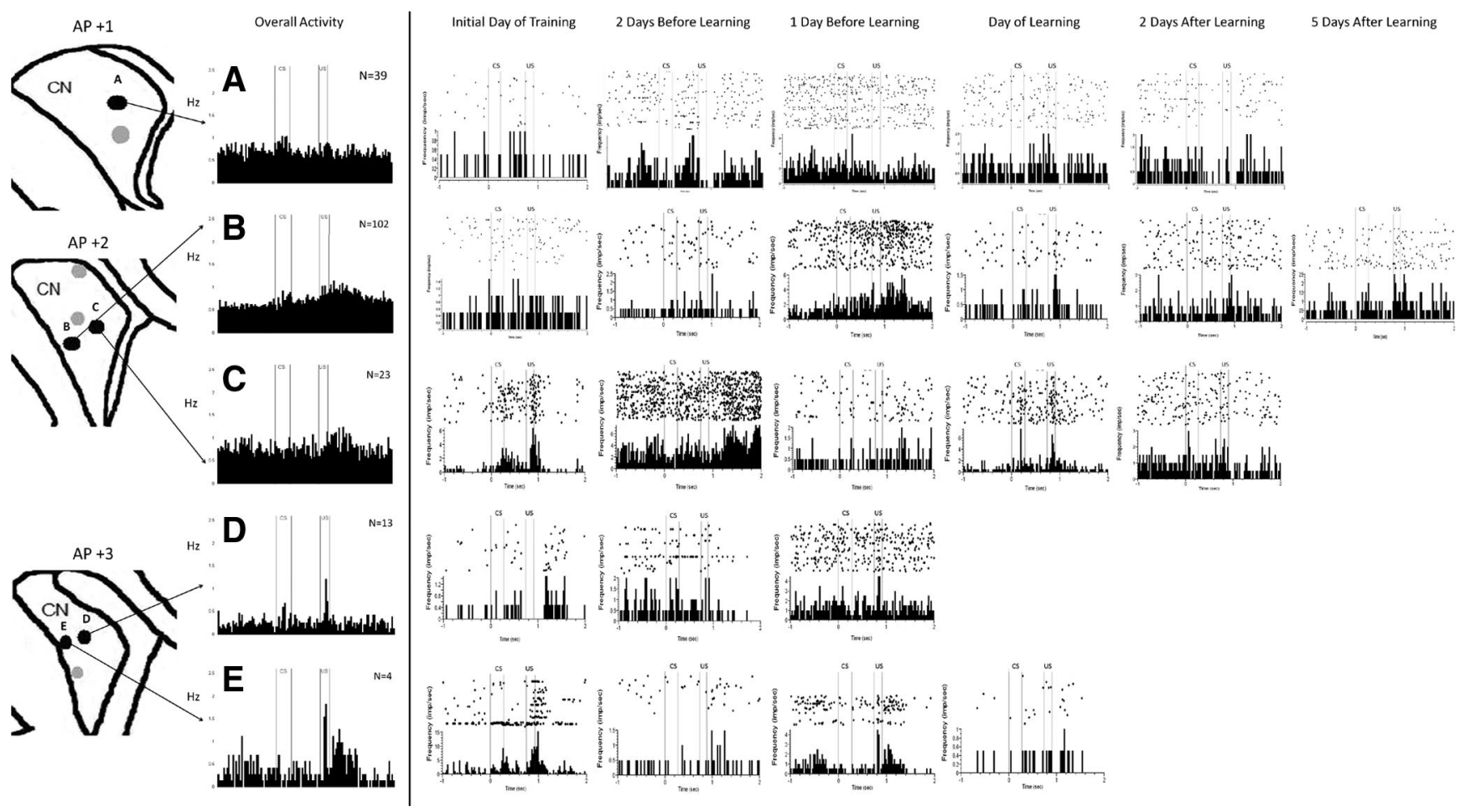

Figure 6. MSNs undergo learning-related changes in discrete locations of the right caudate nucleus. MSNs in the right CN are less responsive to trace EBC than MSNs in the left CN. Region differences can still be seen. Regions C and E show responsiveness beginning on the initial day of training. Clear patterns of responsivity are more difficult to detect in other region of the right CN.

been reported in rabbits with hippocampus lesions (Solomon et al., 1986) or barrel cortex lesions (Galvez et al., 2007). CNlesioned rabbits undergoing pseudoconditioning did not show a higher percentage of alpha blinks than sham-lesioned rabbits, indicating that the increase in the percentage of alpha responses is a learning-related phenomenon. The high percentage of these alpha blinks suggests that the $\mathrm{CN}$ might be playing a role in establishing appropriately timed conditioned responses during trace EBC.

Single-unit recordings taken in vivo from rabbits during training demonstrated that MSNs showed learning-related changes during trace EBC. The changes were greater in the left $\mathrm{CN}$ (contralateral to the trained eye) than in the right (ipsilateral) $\mathrm{CN}$. These hemispheric differences likely arose from the fact that we trained the right eye in these experiments. One change was a significant increase in baseline firing rate in the left $\mathrm{CN}$ during trace $\mathrm{EBC}$ compared with pseudo $\mathrm{EBC}$. This increase in firing rate occurred in the days immediately before learning criterion was met. There was a trend for an increase in baseline firing rate in the right $\mathrm{CN}$ as well. The fact that these neurons became more excitable at baseline before behavioral learning might indicate that the striatum was selecting particular patterns of corticostriatal inputs to potentiate, as has been proposed previously (Schultz et al., 1997).

The second change in MSN firing was a strong increase in firing in response to the CS and US. This activity pattern was fundamentally different from the activity that has been reported in the rabbit $\mathrm{CN}$ during delay EBC (White et al., 1994). White and colleagues reported activity that models the eyeblink, much like the activity previously reported in the hippocampus during delay EBC (Berger et al., 1983). Although MSNs showed responses to the air puff during US trials of pseudoconditioning, there was no response to whisker stimulation during CS trials of pseudoconditioning, indicating that the change in responsivity to the CS was learning related. The changes in firing during learning appeared in critical regions of the head and rostral body of the $\mathrm{CN}$ during the first days of training, well before the rabbit began performing any CRs, suggesting a role in memory formation. Why would different regions of the $\mathrm{CN}$ show different patterns of response during trace $\mathrm{EBC}$ ? The corticostriatal inputs into the $\mathrm{CN}$ are very highly organized, and so $\mathrm{CN}$ activity reflected the activity of the cortical regions that project to it. The $\mathrm{CN}$, probably with help from dopaminergic inputs from the substantia nigra pars compacta, presumably selected which of these corticostriatal inputs to potentiate and which to depress. This selection process could also explain the increase in firing rate seen in certain regions of the left $\mathrm{CN}$ in the days before learning criterion as well as the strong response to the CS and US. It should also be noted that, although MSNs in the right CN did not show as strong responsivity to the CS and US as MSNs in the left CN, it is clear that these neurons were responsive. This finding strengthens the results of the lesion study, in which the medial and ventral $\mathrm{CN}$ had to be lesioned bilaterally to prevent acquisition of trace EBC. In the case of a unilateral lesion, it is possible that the $\mathrm{CN}$ on the opposite side could compensate for the loss. Lesions of the hippocampus and caudal ACC also had to be bilateral to see lesion effects (Moyer et al., 1990; Weible et al., 2000).

TANs were recorded and distinguished from the more numerous MSNs observed in vivo from the rabbit CN. These TANs showed learning-related changes during trace $\mathrm{EBC}$, similar to the results found in primates during delay EBC (Blazquez et al., 2002). TANs showed a pause in response to the air-puff US early in training, with a smaller pause in firing in response to the CS. This pause transferred to the whisker stimulation CS after the rabbit achieved learning criterion. There was also a striking rebound in firing rate that occurred after the CS and during the trace period. This rebound in firing has been observed after delivery of sensory stimuli in primates (Ravel et al., 2003), so it is 
difficult to say whether it is a response to the trace period or a natural neuronal response after a period of inhibition. These recordings are the first example of the pause transferring across a stimulus-free trace period to the onset of the conditioned stimulus. The overall activity of TANs was different from MSNs. Whereas MSNs showed the strongest changes before the rabbit acquired trace $\mathrm{EBC}$, TANs became most responsive to the CS after the association had been made. It is also clear that TANs remained responsive to the CS for at least $9 \mathrm{~d}$ of overtraining, after the point when learning criterion had been reached. This suggests that the CS-US association can be represented by TANs bilaterally in the $\mathrm{CN}$, even after most of the MSNs have stopped responding.

The initial changes in neuronal activity precede the day of acquisition, which is consistent with the fact that pretraining lesions were effective in preventing acquisition of trace EBC. The smallest effective lesion included regions of the $\mathrm{CN}$ that showed the earliest changes during trace EBC. The medial region of rostral body of the CN showed CS and US activity on the very first day of training. This region receives direct input from the ACC, which is a cortical region that also undergoes learning-related changes in neuronal activity during the first days of training and is essential for acquisition of trace EBC (Weible et al., 2000, 2003, 2007). There is also evidence that the perirhinal cortex, which relays output from the hippocampus, projects to multiple regions of the ventral and dorsomedial $\mathrm{CN}$ in the cat (Witter and Groenewegen, 1986). These regions of the $\mathrm{CN}$ also showed significant changes in neuronal activity during trace EBC, especially in the days immediately before learning criterion when CS and US activity begin to appear in the hippocampus (McEchron and Disterhoft, 1997). These changes indicate that the $\mathrm{CN}$ is integrating activity from different cortical regions, as has been proposed previously (Alexander et al., 1986). These changes in the $\mathrm{CN}$ are necessary for the formation of properly timed and executed adaptive CRs, as demonstrated in the lesion study.

A few studies have looked at the role of the basal ganglia in humans for EBC. Patients with Parkinson's disease are not impaired in delay or trace EBC (Sommer et al., 1999), but patients with progressive supranuclear palsy are impaired in both delay and trace EBC (Sommer et al., 2001). These diseases affect the substantia nigra, which provides dopaminergic in-
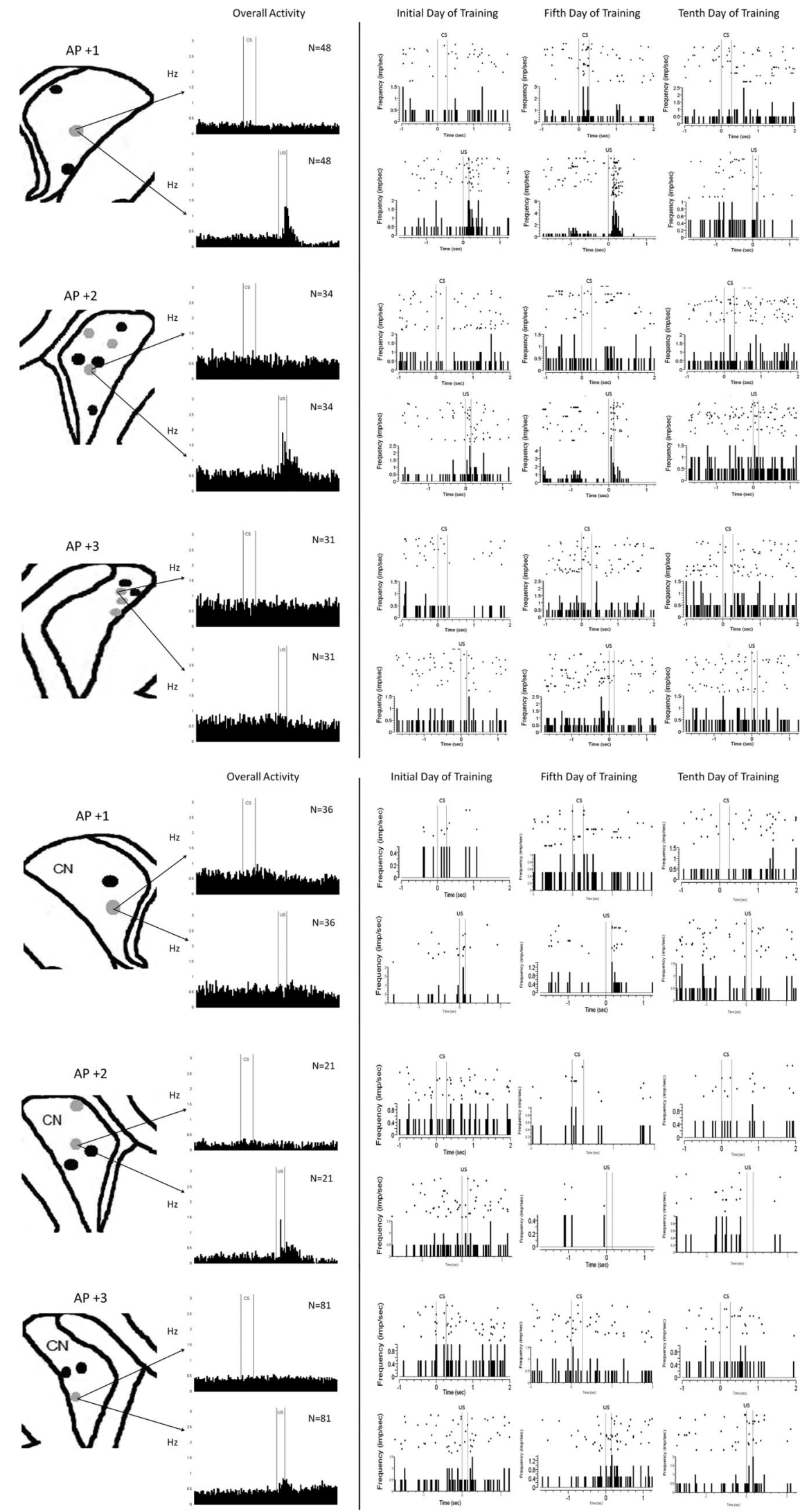

Figure 7. MSNs only respond to the US during pseudoconditioning. Recordings sites during pseudoconditioning (gray dots) from the left and right CN. MSNs are only responsive to the US and not to the CS during pseudoconditioning. MSNs are more responsive in the left $\mathrm{CN}$ than the right $\mathrm{CN}$. 


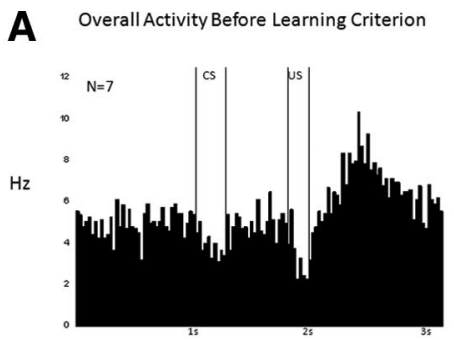

B

Overall Activity After Learning Criterion

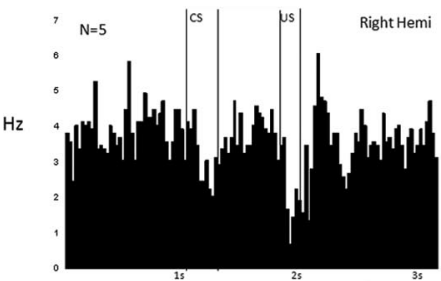

C

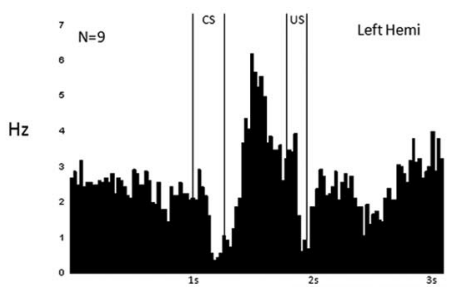

D

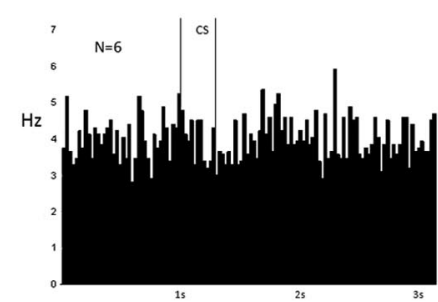

Pseudo EBC: US Trials - Left and Right CN

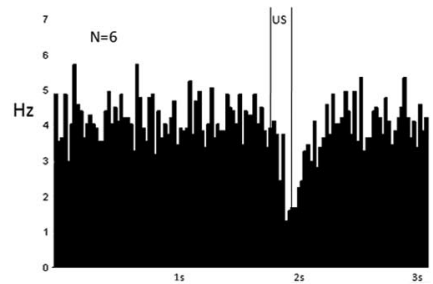

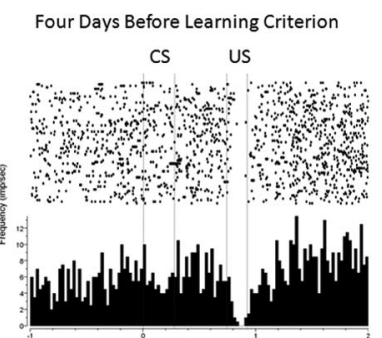

Day of Learning Criterion CS US

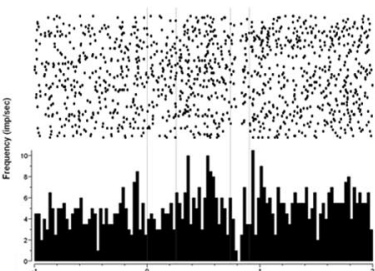

inestis

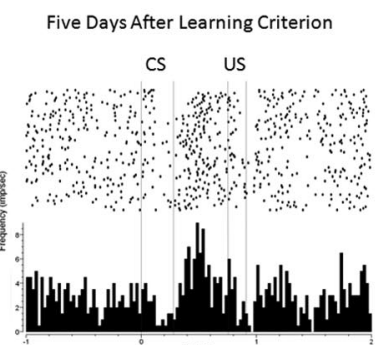

Left Hemisphere

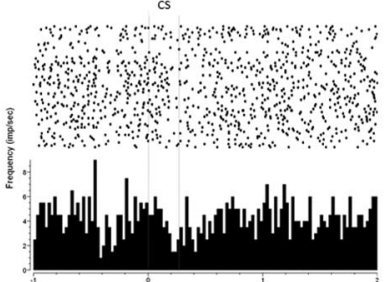

Left Hemisphere

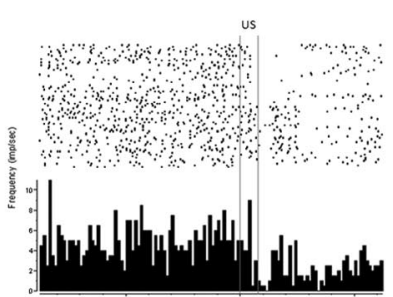

mentes

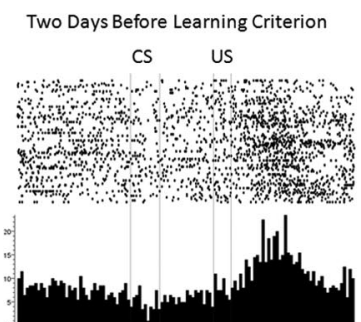

CS US

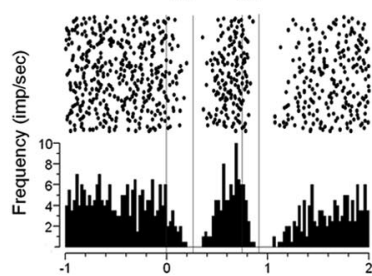

Seven Days After Learning Criterion

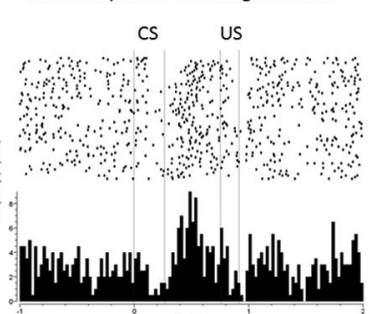

Right Hemisphere

cs

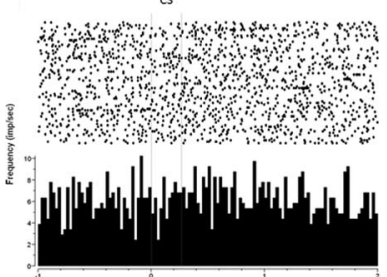

Right Hemisphere

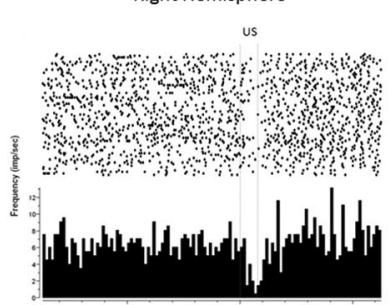

One Day Before Learning Criterion

CS US

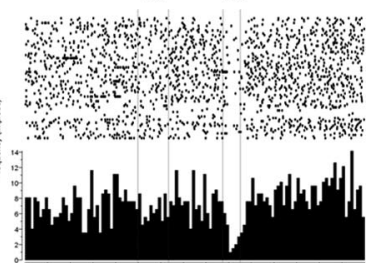

Four Days After Learning Criterion CS US

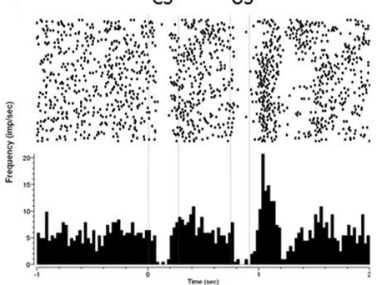

Nine Days After Learning Criterion

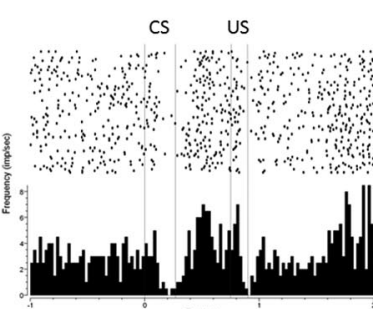

Figure 8. Tonically active neurons in the right and left caudate nucleus during trace and pseudo EBC. A, Overall activity from seven TANs recorded from the right CN before learning criterion, followed by raster plots from individual TANs 4,2 , and $1 \mathrm{~d}$ before learning criterion. There is a very small pause in tonic firing in response to the $C S$ and a larger pause and rebound in response to the US. $\boldsymbol{B}$, Overall activity from five TANs recorded from the right $\mathrm{CN}$ on and after the day of learning criterion, followed by raster plots from individual TANs on the day of learning criterion and 2 and $4 \mathrm{~d}$ after learning criterion. A pause in response to the $\mathrm{CS}$ is seen in some, but not all, TANs in the right $\mathrm{CN}$ after learning criterion. $\mathrm{C}$, Overall activity for nine TANs recorded from the left $\mathrm{CN}$ after learning criterion, followed by raster plots from individual TANs 5, 7, and $9 \mathrm{~d}$ after learning criterion. All TANs recorded from the left CN paused their firing in response to the CS and increased their firing during the trace period after learning criterion. D. TANs in the left and right $\mathrm{CN}$ do not respond to whiskers stimulation during $\mathrm{CS}$ trials of pseudoconditioning. TANs in both the left and right $\mathrm{CN}$ pause their tonic firing in response to the air puff during US trials of pseudoconditioning.

put into the striatum. Huntington's disease, which affects the MSNs in the striatum directly, is a more suitable comparison with the $\mathrm{CN}$ lesions made in these experiments. A study by WoodruffPak and Papka (1996) tested patients with Huntington's disease in simple delay EBC and demonstrated that they had normal learning but abnormal timing of CRs. Their results complement our findings in trace conditioning, in which CN-lesioned rabbits could not perform adaptive CRs but showed a high percentage of alpha responses.

Together, our findings demonstrate that the $\mathrm{CN}$ may be as important to the acquisition of trace $\mathrm{EBC}$ as the hippocampus. Learning-related changes occur in parallel in both of these struc- 
tures, and neither structure is able to fully compensate for the loss of the other in this forebrain-dependent learning task. We hypothesize that, although the hippocampus is necessary for associating the CS with the US during trace conditioning, it is unable to select an appropriate motor response to the CS. The basal ganglia could serve this role given its strong connectivity with motor cortices and brainstem nuclei, as well as its established role in motor planning and coordination.

\section{References}

Alexander GE, DeLong MR, Strick PL (1986) Parallel organization of functionally segregated circuits linking basal ganglia and cortex. Annu Rev Neurosci 9:357-381.

Aosaki T, Tsubokawa H, Ishida A, Watanabe K, Graybiel AM, Kimura M (1994) Responses of tonically active neurons in the primate's striatum undergo systemic changes during behavioral sensorimotor conditioning. J Neurosci 14:3969-3984.

Balleine BW, Liljeholm M, Ostlund SB (2009) The integrative function of the basal ganglia in instrumental conditioning. Behav Brain Res 199:43-52.

Berger TW, Rinaldi PC, Weisz DJ, Thompson RF (1983) Single-unit analysis of different hippocampal cell types during classical conditioning of rabbit nictitating membrane response. J Neurophysiol 50:1197-1219.

Blazquez PM, Fujii N, Kojima J, Graybiel AM (2002) A network representation of response probability in the striatum. Neuron 33:973-982.

Broadbent NJ, Squire LR, Clark RE (2007) Rats depend on habit memory for discrimination learning and retention. Learn Mem 14:145-151.

Christian KM, Thompson RF (2003) Neural substrates of eyeblink conditioning: acquisition and retention. Learn Mem 10:427-455.

Das S, Weiss C, Disterhoft JF (2001) Eyeblink conditioning in the rabbit (Oryctolagus cuniculus) with stimulation of the mystacial vibrissae as a conditioned stimulus. Behav Neurosci 115:731-736.

Galvez R, Weiss C, Weible AP, Disterhoft JF (2006) Vibrissa-signaled eyeblink conditioning induces somatosensory cortical plasticity. J Neurosci 26:6062-6068.

Galvez R, Weible AP, Disterhoft JF (2007) Cortical barrel lesions impair whisker-CS trace eyeblink conditioning. Learn Mem 14:94-100.

Girgis M, Shih-Chang W (1981) A new stereotaxic atlas of the rabbit brain. St. Louis: Green.

Gormezano I, Schneiderman N, Deaux E, Fuentes I (1962) Nictitating membrane: classical conditioning and extension in the albino rabbit. Science 138:33-34.

Hoffer ZS, Alloway KD (2001) Organization of corticostriatal projections from the vibrissal representation in the primary motor and somatosensory cortical areas of rodents. J Comp Neurol 439:87-103.

McEchron MD, Disterhoft JF (1997) Sequence of single unit changes in CA1 hippocampus during acquisition of trace eyeblink conditioned responses. J Neurophysiol 78:1030-1044.
Moyer JR Jr, Deyo RA, Disterhoft JF (1990) Hippocampectomy disrupts trace eye-blink conditioning in rabbits. Behav Neurosci 104:243-252.

Packard MG (2009) Exhumed from thought: basal ganglia and response learning in the plus maze. Behav Brain Res 199:24-31.

Packard MG, Knowlton BJ (2002) Learning and memory functions of the basal ganglia. Annu Rev Neurosci 25:563-593.

Parent M, Parent A (2006) Single-axon tracing study of corticostriatal projections arising from primary motor cortex in primates. J Comp Neurol 496:202-213.

Powell DA, Mankowski D, Buchanan SL (1978) Concomitant heart rate and corneoretinal potential conditioning in the rabbit (Orytolagus cuniculus): effects of caudate lesions. Physiol Behav 20:143-150.

Ravel S, Legallet E, Apicella P (2003) Responses of tonically active neurons in the monkey striatum discriminate between motivationally opposing stimuli. J Neurosci 23:8489-8497.

Schultz W, Dayan P, Montague PR (1997) A neural substrate of prediction and reward. Science 275:1593-1599.

Solomon PR, Vander Schaaf ER, Thompson RF, Weisz DJ (1986) Hippocampus and trace conditioning of the rabbit's classically conditioned nictitating membrane responses. Behav Neurosci 100:729-744.

Sommer M, Grafman J, Clark K, Hallett M (1999) Learning in Parkinson's disease: eyeblink conditioning, declarative learning, and procedural learning. J Neurol Neurosurg Psychiatry 67:27-34.

Sommer M, Grafman J, Litvan I, Hallett M (2001) Impairment of eyeblink classical conditioning in progressive supranuclear palsy. Mov Disord 16:240-251.

Weible AP, McEchron MD, Disterhoft JF (2000) Cortical involvement in acquisition and extinction of trace eyeblink conditioning. Behav Neurosci 114:1058-1067.

Weible AP, Weiss C, Disterhoft JF (2003) Activity profiles of single neurons in the caudal cingulate cortex during trace eyeblink conditioning in the rabbit. J Neurophysiol 90:599-612.

Weible AP, Weiss C, Disterhoft JF (2007) Connections of the caudal anterior cingulate cortex in rabbit: neural circuitry participating in the acquisition of trace eyeblink conditioning. Neuroscience 145:288-302.

White IM, Miller DP, White W, Dike GL, Rebec GV, Steinmetz JE (1994) Neuronal activity in rabbit neostriatum during classical eyelid conditioning. Exp Brain Res 99:179-190.

White NM (2009) Some highlights of research on the effect of caudate nucleus lesions over the past 200 years. Behav Brain Res 199:3-23.

Witter MP, Groenewegen HJ (1986) Connections of the parahippocampal cortex in the cat. IV. Subcortical efferents. J Comp Neurol 252:51-77.

Woodruff-Pak DS, Papka M (1996) Huntington's disease and eyeblink classical conditioning: normal learning but abnormal timing. J Int Neuropsychol Soc 2:323-334.

Woodruff-Pak DS, Lavond DG, Thompson RF (1985) Trace conditioning: abolished by cerebellar nuclear lesions but not lateral cerebellar cortex aspirations. Brain Res 348:249-260. 